\title{
Source flash $X$ compacte impulsionnelle à haut taux de répétition
}

\author{
E. Robert, C. Cachoncinlle, A. Khacef, R. Viladrosa, J.M. Pouvesle, C.B. Collins* \\ et F. Davanloo* \\ GREMI, Université d'Orléans, BP. 6759, 45067 Orléans cedex 2, France \\ * Center for Quantum Electronics, University of Texas at Dallas, P.O. Box 830688 , \\ Richardson, Texas 75083-0688, U.S.A.
}

\begin{abstract}
X}$ ray device producing strong $X$-ray doses in nanosecond pulses at high repetition rate. Doses up to 2 rad, at the output window, of $10 \mathrm{keV}$ centered $\mathrm{X}$-rays are emitted either along a $10 \mathrm{~cm}$ long filament or as a spot of less than $300 \mu \mathrm{m}$ in diameter depending on the electrode configuration. The use of a rotating anode and of an external electrode ajustment have enhanced the reliability of the device which can now operate for hours at $20 \mathrm{~Hz}$ at constant output power.
\end{abstract}

Nous avons développé et optimisé une source flash $X$ [1] présentant des caractéristiques intéressantes dans des domaines d'application variés tels que la radiographie éclair, l'ombroscopie, la physico-chimie des plasmas.

Le fonctionnement du flash est basé sur la production d'une décharge de très courte durée (qq. dizaines de nanosecondes) et de forte énergie (qq. joules) entre des électrodes placées sous vide résiduel. La mise en forme de l'impulsion haute tension est réalisée au moyen d'une ligne Blumlein (capacité $2 \times 4.3 \mathrm{nF}$ ) commutée par un thyratron (EG\&G HY 5313). L'ensemble ligne-élément de commutation est placé dans un container métallique contenant de l'huile spéciale haute tension et jouant le rôle de cage de Faraday. Les dimensions du système sont de $1.4 \times 0.3 \times 0.3 \mathrm{~m}^{3}$. La ligne Blumlein est chargée de manière impulsionnelle à l'aide d'une alimentation à découpage haute tension (40 kV, Converter Power RSC-3000) compacte $\left(0.4 \times 0.4 \times 0.2 \mathrm{~m}^{3}\right)$.

L'étude de la dose émise en fonction de la pression résiduelle dans la diode $\mathrm{X}$, de la tension de charge, de l'espace entre les électrodes, de la géométrie et du matériau des électrodes a permis de déterminer les points de fonctionnement optimaux du système. Ces paramètres facilement ajustables permettent d'obtenir des impulsions X d'intensité et de largeur temporelle variables. Deux géométries d'électrodes ont été exploitées. En configuration lame graphite-rondin $W$, l'émission $X$ est homogène sur un filament de 10 
$\mathrm{cm}$ de long et $1.5 \mathrm{~mm}$ de diamètre. En configuration pointe graphite $(\varnothing \sim 300 \mu \mathrm{m})$ rondin W, la taille de la source mesurée par sténopée est inférieure à $300 \mu \mathrm{m}$.

Les principales caractéristiques de la source, à son stade actuel de développement, sont les suivantes:

Spectre émis:

la plus grande partie est émise dans les raies caractéristiques du matériau de l'anode; W (7-12 $\mathrm{keV})$, Mo $(17-20 \mathrm{keV}), \mathrm{Cu}(8-9 \mathrm{keV})$ sur un faible fond continu de bremsstrahlung

Domaine d'énergie des photons $X$ : de 5 à $50 \mathrm{keV}$

Energie rayommée:

de l'ordre du mJ dans $4 \pi$ stéradian

Durée de l'impulsiom:

ajustable de 3 à 50 ns

Dose par tir:

jusqu'à 2 rad à la fenêtre de sortie et 12 mrad à 1 mètre de la source

Débit de dose:

supérieur à $2 \mathrm{krad} / \mathrm{mn}$ à $30 \mathrm{kV}$ et à $50 \mathrm{~Hz}$

Toux de répétition:

du monocoup à $50 \mathrm{~Hz}$

Nombre de photons $X$ émis par tir: de l'ordre de $10^{12}$

L'asservissement de l'anode à l'axe d'un moteur assure une rotation et une translation par rapport à la cathode de la région émettrice du rayonnement $X$. La réalisation de cette anode tournante et d'un système externe de réglage de l'espacement des électrodes a considérablement augmenté la fiabilité du dispositif et permet un fonctionnement en routine $(10 \mathrm{~Hz}$ pendant plusieurs jours) sans modification importante à la fois de la dose émise (fluctuations autour de la dose moyenne $<15 \%$ sur plusieurs heures) et de la largeur temporelle de l'impulsion.

Cette nouvelle source a montré son efficacité dans la production de plasmas de gaz rares [2], l'étude résolue en temps de ces plasmas, la radiographie éclair (ombroscopie de linteraction faisceau laser excimère-cibles liquides) [3].

Ce travail a bénéficié des supports financiers de la DRET et du Conseil Régional du Centre

[1] Pouvesle J.M., Cachoncinlle C., Robert E., Viladrosa R., Collins C.B. et Davanloo F., Rev. Sci. Instrum. 64 (8) 1993

[2] Robert E., Khacef A., Cachoncinlle C., Viladrosa R., Pouvesle J M., Collins C.B. et Davanloo F., "Energetic high repetition rate flash $\mathrm{X}$-ray source: application to UV-VUV fluorescence studies in high pressure gases", XXI ICPIG, Bochum 1924 September 1993, Vol. invited papers pp.237-244.

[3] Cachoncinlle C., Challine I., Jobard F., Robert E. et Pouvesle J.M., "Approche de linteraction laser UV-cible liquide par ombroscopie X", annales de physique UVX94 (ce volume). 